\title{
Die Bewegungstrajektorie einer Gasblase im stark durchlüfteten Bereich von Strahlapparaten mit unterschiedlich geformten Düsen
}

\author{
Wladimir Jurjewitsch Lobov, Alexandr Viktorovitsch Sugak, Grigori Michailowitsch Gontscharov
}

Durch Lösen der Newtonschen Gleichung unter Zuhilfenahme eines Ansatzes über die Geschwindigkeitsverteilung eines turbulenten Strahls unter der Flüssigkeitsoberfläche wird eine Methodik zur Berechnung der aktiv durchlüfteten Zone in Strahlapparaten vorgeschlagen.

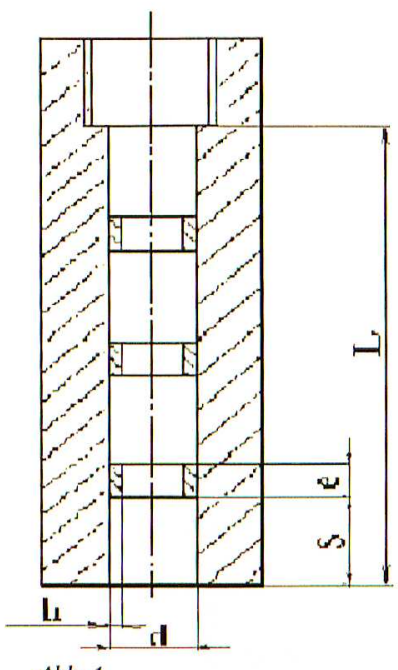

Das Entwicklungstempo der verschiedensten Bereiche der Industrie erfordern eine ständige Verbesserung der konstruktiven Ausführung von Apparaturen fuir den Stoffaustausch. Diese Aufgabe bezieht sich ebenso auf Strahlapparate, die dank ihrer Vorteile in letzter Zeit eine immer weitere Verbreitung finden: eine hohe Auflösungsgeschwindigkeit von Gasen in Flüssigkeiten, Energieeinsparung und die Einfachheit der Konstruktion. Die Vervollkommnung der Konstruktion der Strahlapparate wird in der vorliegenden Arbeit durch den Einsatz von Düsen mit unterschiedlichen konstruktiven Einsätzen, wie sie in Abb. 1 dargestellt sind, erreicht. Dadurch wird die Oberfläche des Strahls aufgerauht. Die Parameter der Diisenoberflächen sind in Tab. 1 dargestellt.

In der Literatur wurde die Frage über die Abmessungen der aktiven Zone bisher rein empirisch untersucht $[1$, 2]. In der vorliegenden Arbeit soll gezeigt werden, daß es möglich ist, die Größe der aktiv belüfteten Zone theoretisch durch das Lösen der Newtonschen Gleichung unter Beriicksichtigung der Geschwindigkeitsverteilung in einem eingetauchten, turbulenten, axialsymmetrischen Strahl zu bestimmen.

\begin{tabular}{|c|c|c|}
\hline Die Charakteristik der & $\mathrm{e} / \mathrm{s}$ & $0.13-1$ \\
\cline { 2 - 3 } Düsenoberfläche & $\mathrm{h} / \mathrm{d}$ & $0.08-0.16$ \\
\hline
\end{tabular}

Trkefţlgenden nehmen wir eine Reihe von Vereinfachungen vor:

1. Es wird angenommen, dab der Anteil der Gasphase in der aktiv durchlüftetenn Zone gering ist und ca. 30\% beträgt. Das wurde auch experimentell bestätigt. Unter diesen Umständen üben die Gasblasen nur einen unwesentlichen Einfluß auf die Geschwindigkeitsverteilung in der flüssigen Phase aus. Somit läßt sich die Geschwindigkeitsverteilung als definiert ansehen, sie stimmt mit der Geschwindigkeitsverteilung in einem eingetauchten turbulenten Strahl überein [3].

2. Im Moment des Eintauchens des Strahls in die Flïssigkeit nimmt die Größe der Gasbläschen rasch zu, [1] und deren Durchmesser beträgt im Verlaufe der Bewegung in der Flïssigkeit ungefähr $3 \mathrm{~mm}$. Wie unsere Messungen am System „Wasser-Luft“ mit unterschiedlichen Geschwindigkeiten zeigten, hängt der Durchmesser nur unwesentlich von der Strahlgeschwindigkeit ab. Im weiteren wird angenommen, daß die Gasblasen einen konstanten Durchmesser vom Moment des Eintritts in die Flüssigkeit an bis zum Austritt an die Oberfläche aufweisen.

Somit wird die Aufgabe auf die Betrachtung der Bewegung einer einzelnen Gasblase in einem bekannten Fluissigkeitsstrom zurückgefuihrt. Für die Berechnun-

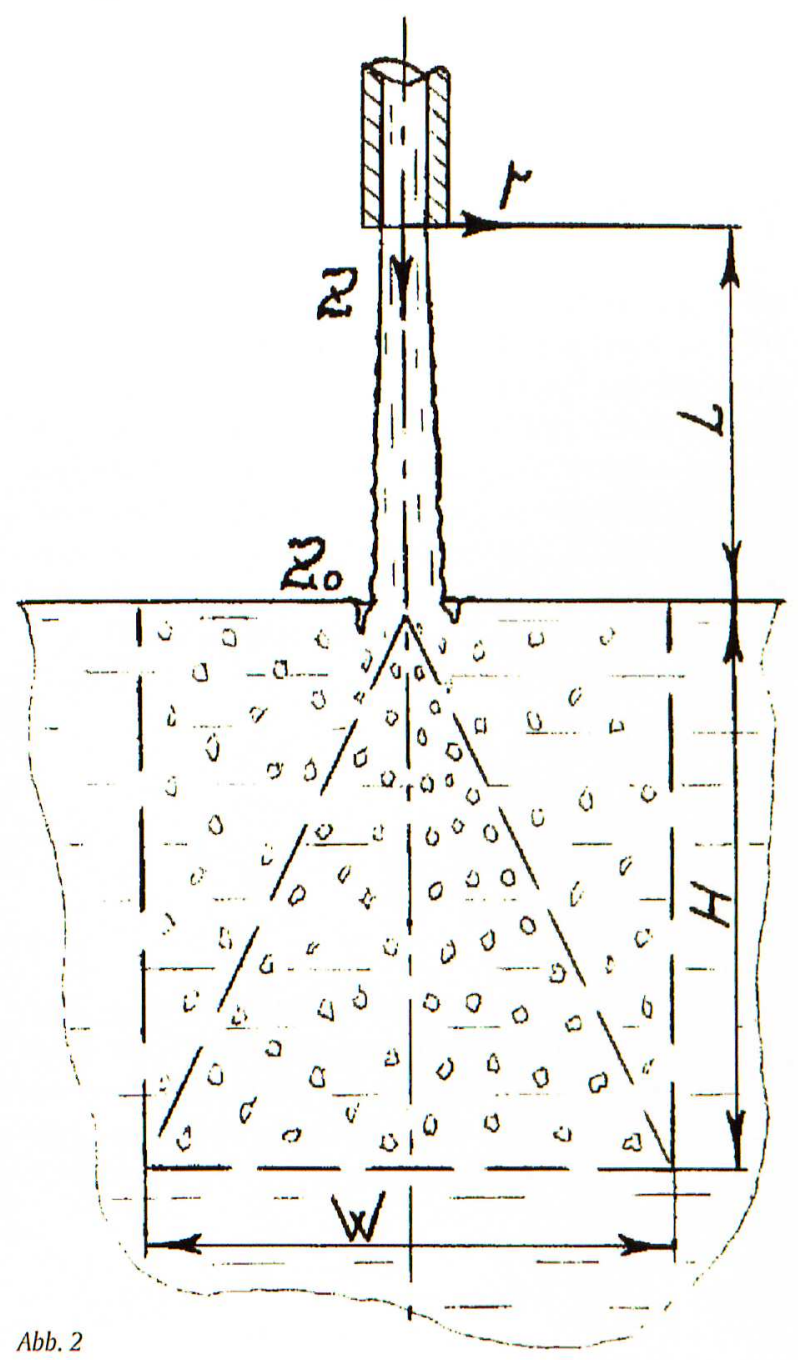


gen wurde das zylindrische Koordinatensystem gewählt, wobei die z-Achse entlang der Strahlachse verläuft (Abb. 2).

Der eingetauchte Strahl wurde mit Hilfe der NavierStokesschen Gleichung berechnet. Die turbulente Strömung wurde mit der halbempirischen Prandtl-Theorie beruicksichtigt [4]. Die genauen Lösungswege der Gleichungen sind in einer Reihe von Literaturquellen zu finden $[3,4]$, wir beschränken uns daher auf die Darstellung des Ergebnisses für die Verteilungen der Longitudinal $[u]$ - und der Transversalgeschwindigkeit $[v]$ :

$$
\begin{aligned}
& u=\frac{3}{8 \pi \sigma} \sqrt{\frac{J_{0}}{\rho}} \frac{1}{z} \frac{1}{\left[1+\frac{3}{64 \pi \sigma^{2}}\left(\frac{r}{z}\right)^{2}\right]^{2}}, \\
& v=\frac{3}{16 \pi \sigma} \sqrt{\frac{J_{0}}{\rho}} \frac{r}{z^{2}} \frac{\left[1-\frac{3}{64 \pi \sigma^{2}}\left(\frac{r}{z}\right)^{2}\right]}{\left[1+\frac{3}{64 \pi \sigma^{2}}\left(\frac{r}{z}\right)^{2}\right]^{2}} .
\end{aligned}
$$

Der Parameter s ist eine empirische Konstante. Für einen runden Strahl beträgt der Parameter $\sigma=0,021$ [3]. Die Flußdichte $J_{0}$ des Strahls läßt sich mit der Ausfließgeschwindigkeit der Fluissigkeit aus der Düse $V_{0}$ und dem Durchmesser der Düse $d$ iiber folgende Beziehung darstellen:

$$
\sqrt{\frac{\mathrm{J}_{0}}{\rho}}=\frac{\beta}{16 \sigma} \mathrm{V}_{0} \mathrm{~d}
$$

wo $\beta$ - ein empirischer Koeffizient ist. Für eine runde Öffnung beträgt $\beta \approx 1.5$ [5].

Nun muß die Lage der Düse auf der Achse bestimmt werden. Dafür wird angenommen, daß die Longitudinalgeschwindigkeit des Strahls $u$ beim Eintritt in die Fliissigkeit mit der Longitudinalgeschwindigkeit im eingetauchten Strahl übereinstimmt. Wenn man annimmt, daß sich die Diise im Koordinatenursprung befindet, kann die Koordinate für die Fluissigkeitsoberfläche $z_{0}$ wie folgt gefunden werden:

$$
\mathrm{z}_{0}=2 \mathrm{Ad}
$$

Wo

$$
A=\frac{3 \beta}{256 \pi \sigma^{2}} \text {. }
$$

Im folgenden werden die Newtonschen Bewegungsgleichungen, die für die Bewegung einer Gasblase in einem bestimmten Fluß gelten, aufgeschrieben. Auf diese Blase wirkt eine Kraft durch die strömende Flüssigkeit, eine Auftriebskraft und eine Widerstandskraft:

$$
\frac{\mathrm{d}^{2} \mathrm{r}}{\mathrm{dt}^{2}}=\left(\frac{\mathrm{dr}}{\mathrm{dt}} \nabla\right) \mathrm{v}(\mathrm{r})-\mathrm{g}+\frac{9 \mathrm{v}}{\mathrm{R}^{2}}\left(\frac{\mathrm{dr}}{\mathrm{dt}}-\mathrm{v}(\mathrm{r})\right)
$$

wobei der Nabla-Operator durch folgende Beziehung bestimmt wird:

$$
\nabla=\mathrm{i} \frac{\partial}{\partial x}+\mathrm{j} \frac{\partial}{\partial y}+\mathrm{k} \frac{\partial}{\partial z}
$$

$i, j$ und $k$ - sind die Richtungsvektoren für die $x, y$ und $z$ - Achse.

Die Gleichung (4) kann nicht analytisch berechnet werden, daher muß sie numerisch in den kartesischen Koordinaten $x, y, z$ bestimmt werden. Die Geschwindigkeitsverteilung in diesen Koordinaten kann mit den entsprechenden zylindrischen Geschwindigkeiten $u$ und $v$ aus Gleichung (1) wie folgt gekoppelt werden:

$v_{x}=v \frac{x}{r}, v_{y}=v \frac{y}{r}, v_{z}=u$.

Aus Bequemlichkeitsgründen können dimensionslose Koordinaten $X, Y, Z$ und die dimensionslose Zeit $\tau$ eingefuihrt werden:

$X=\frac{x}{d}, \quad Y=\frac{y}{d}, \quad Z=\frac{z}{d}, \quad \tau=t \frac{V_{0}}{d}$.

Die Aufgabe ist zylindersymmetrisch, daher läßt sich eine Gleichung ausschließen. Wenn man $Y(0)=0$ und $d Y$ / $d t=0$ setzt, läßt sich die Aufgabe mit Hilfe von zwei Differentialgleichungen zweiter Ordnung beschreiben. Die erforderlichen Ableitungen in Gleichung (4) können mit Hilfe von Gleichung (1) berechnet werden und wir erhalten folgende Gleichungen:

$$
\begin{aligned}
& \frac{d^{2} X}{d \tau^{2}}=A\left\{\frac{\left[Z^{2}\left(1-\Phi^{4}\right)-2 a^{2} X^{2}\left(3-\Phi^{2}\right)\right] \frac{d X}{d \tau}-2 X Z\left(1-3 \Phi^{2}\right) \frac{d Z}{d \tau}}{Z^{4}\left(1+\Phi^{2}\right)^{3}}\right\}- \\
& \frac{9 v d}{V_{0} R^{2}}\left[A \frac{X\left(1-\Phi^{2}\right)}{Z^{2}\left(1+\Phi^{2}\right)^{2}}-\frac{d X}{d \tau}\right] \\
& \frac{d^{2} Z}{d \tau^{2}}=2 A\left\{\frac{-Z^{2}\left(1-3 \Phi^{2}\right) \frac{d Z}{d \tau}-4 a^{2} X Z \frac{d X}{d \tau}}{Z^{4}\left(1+\Phi^{2}\right)^{3}}\right\}-\frac{g d}{V_{0}^{2}}-\frac{9 v d}{V_{0} R^{2}}\left[\frac{2 A}{Z\left(1+\Phi^{2}\right)^{2}}-\frac{d Z}{d \tau}\right]
\end{aligned}
$$

Es wurden folgende Bezeichnungen eingefuihrt:

$$
\mathrm{a}=\frac{\sqrt{3 / \pi}}{8 \sigma}, \quad \Phi=\mathrm{a} \frac{\mathrm{X}}{\mathrm{Z}}, \quad \mathrm{A}=\frac{3 \beta}{256 \pi \sigma^{2}} .
$$

Das Gleichungssystem (8) besitzt eine eindeutige Lösung, wenn die Koordinaten der Gasblase zum Zeitpunkt Null und die Geschwindigkeitskomponenten vorgegeben werden. Unter der Annahme, daß sich bei $\tau=0$ die Gasblase an der Oberfläche der Flüssigkeit irgendwo innerhalb des Strahls befindet, gilt:

$X(0)=X_{0},\left(-0.5 \leq X_{0} \leq 0.5\right), \quad Z(0)=\frac{Z_{0}}{d}$.

$z_{0}$ ist durch die Gleichung (3) bestimmt, und die Wahl der Koordinate $X_{0}$ ergibt sich durch die Möglichkeit, die 
Lage des Gasbläschens im Zentrum des Strahls $\left(X_{0}=0\right)$ und an dessen Rand $\left(X_{0}=0,5\right)$ sowie in einer beliebigen Zwischenposition verfolgen zu können. Weitaus schlechter definiert sind die Komponenten für die Anfangsgeschwindigkeit der Gasblase. Eindeutig ist lediglich, daß diese Geschwindigkeit ungefähr gleich $V_{0}$ sein sollte. Somit läßt sich für die Anfangswerte der dimensionslosen Geschwindigkeiten die folgende Beziehung schreiben:

$$
\left.\frac{\mathrm{dX}}{\mathrm{d} \tau}\right|_{\tau=0}=0,\left.\quad \frac{\mathrm{dZ}}{\mathrm{d} \tau}\right|_{\tau=0}=\mathrm{q},
$$

wobei der Parameter $q \sim 1$.

Das Gleichungssystem für die Bewegung der Gasblase (8) wurde numerisch mit dem Runge - Kutta-Verfahren auf einem IBM PC gelöst. Als Endpunkt für die Berechnungen wurde der Moment des Austritts der Gasblase durch die Oberfläche festgelegt, das heißt, wenn folgende Bedingung erfüllt war:

$$
Z\left(\tau_{\text {end }}\right)=Z(0)
$$

wobei $\tau_{\text {end }}$ - der Endpunkt der Berechnungen ist.

Die Ergebnisse dieser Berechnungen sind in Abb. 3 dargestellt.

Der absolute Wert für die Eindringtiefe $H$ ist wie folgt bestimmt:

$$
\mathrm{H}=\mathrm{d} \cdot\left[\max (\mathrm{Z})-\mathrm{Z}_{0}\right] \quad \text { при } \mathrm{X}_{0}= \pm 0,5 .
$$

Als Fackelbreite $W$ wird die doppelte Koordinate des Austritts der Gasblase an die Oberfläche angenommen:

$$
\mathrm{W}=2 \mathrm{dX}\left(\tau_{\text {end }}\right)+1 \text {. }
$$

wobei l eine charakteristische Größe für die pulsierende Bewegung der Blase darstellt. Es wurde in jedem Fall fuir $/$ ein und derselbe Wert gefunden:

$$
1 \approx 5,9 \mathrm{~cm}, \quad \gamma \approx 4,9 \text {. }
$$

Hier ist $\mathrm{g}=1 / d$ ein universeller Parameter, der die turbulente Bewegung charakterisiert.

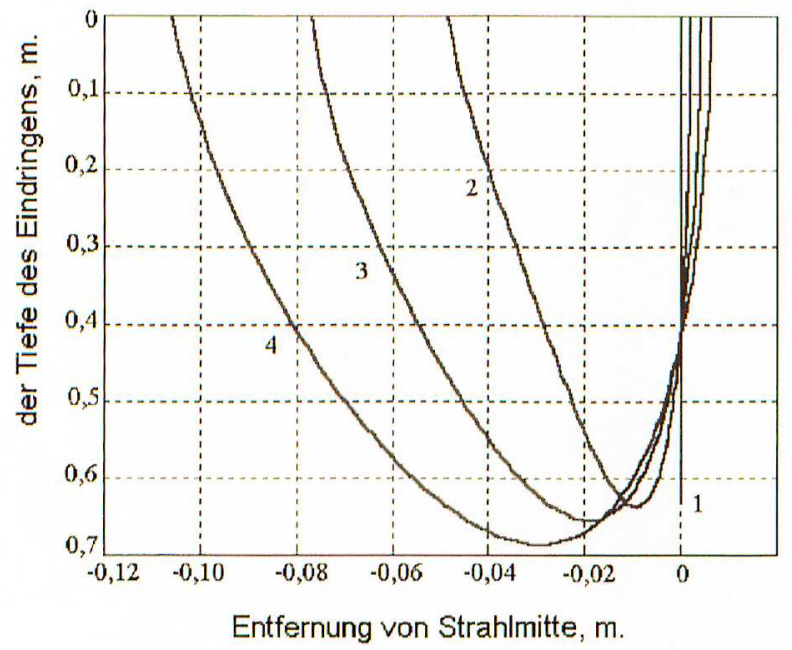

Im Experiment wurde gefunden, daß der Umfang der Fackel von der Länge des Strahls $L$ abhängt, der von 20 bis zu $520 \mathrm{~mm}$ variierte. Es ist offensichtlich, dab dieser Faktor die Anfangsgeschwindigkeit der Gasblase beeinflußt, welche als lineare Abhängigkeit zwischen der Geschwindigkeit $V$ und der Durchflußmenge $M$ dargestellt werden kann:

$\mathrm{V}=\mathrm{B}+\mathrm{C} \cdot \mathrm{M}$.

Hierbei müssen $B$ und $C$ als empirische Konstanten angesehen werden, die als lineare Funktionen von der Strahllänge $L$ abhängen:

$\mathrm{B}=\mathrm{b}_{1}+\mathrm{b}_{2} \cdot \mathrm{L}, \quad \mathrm{C}=\mathrm{c}_{1}+\mathrm{c}_{2} \cdot \mathrm{L}$.

Demzufolge kann die Fackel, die durch den einfallenden Strahl gebildet wird, mit vier Parametern $b_{1}, b_{2}, c_{1}, c_{2}$ beschrieben werden. Die Werte für diese Parameter für verschiedene Düsenformen sind in Tabelle 2 dargestellt. In Abb. 4 sind die Ergebnisse der Berechnungen und der experimentellen Messungen für die Breite und die Höhe der Fackel für die Düse 1 in Abhängigkeit von der Strahllänge dargestellt. Die Abweichung der berechneten Kurven vom Experiment ist geringer als $5 \%$.

\begin{tabular}{|c|c|c|c|c|c|c|}
\hline Düse & $\mathrm{e} / \mathrm{s}$ & $\mathrm{h} / \mathrm{d}$ & $\mathrm{b}_{1}$ & $\mathrm{~b}_{2}$ & $\mathrm{c}_{1}$ & $\mathrm{c}_{2}$ \\
\hline 1 & $\geq 0,4$ & $<0,1$ & 6,26 & $-11,46$ & 3,51 & 5,75 \\
\hline 2 & $<0,4$ & $<0,1$ & 3,75 & $-1,93$ & 4,41 & $-4,16$ \\
\hline 3 & $\geq 0,4$ & $\geq 0,1$ & 3,71 & $-6,73$ & 9,93 & $-1,83$ \\
\hline 4 & $<0,4$ & $\geq 0,1$ & 3,64 & $-3,28$ & 4,80 & $-2,79$ \\
\hline
\end{tabular}

Tabelle 2

Die Modellierung der aktiv durchliifteten Zone erlaubt es, Formeln für die Berechnung von solchen wichtigen Parametern eines Zweiphasensystems zu bestimmen, wie der Gasgehalt und der mittlere Durchmesser der Gasblasen. Im Endeffekt läßt sich die Kontaktoberfläche der Phasen bei der Durchlüftung mit einem Strahlapparat bestimmen. Das ist notwendig, um Berechnungen zum Stoffaustausch in Strahlapparaten durchfuihren zu können.

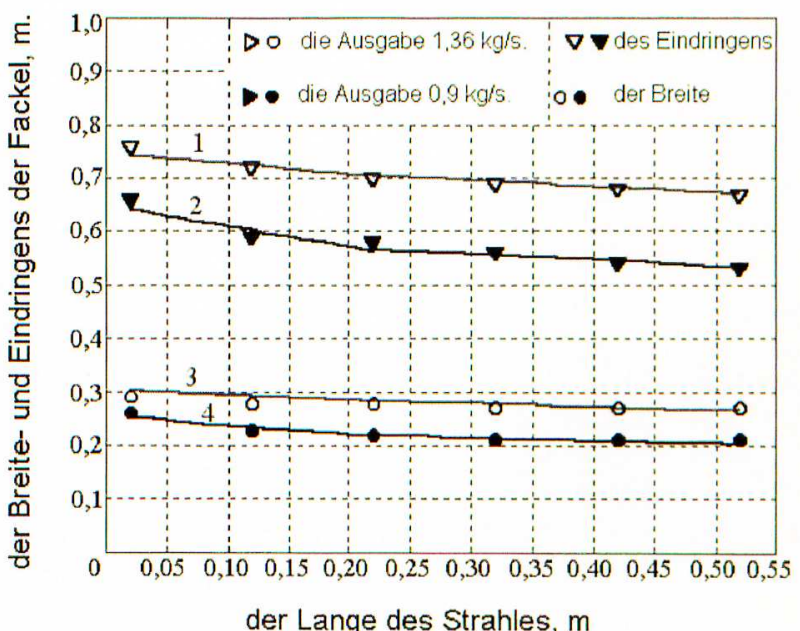

der Lange des Strahles, m 


\section{Schlußfolgerungen}

1. Für die Bestimmung der Bewegungstrajektorie einer Gasblase im aktiv durchluifteten Bereich wurde der Ansatz für die Geschwindigkeitsverteilung in einem eingetauchten turbulenten axialsymmetrischen Strahl verwendet.

2. Vergleichende experimentelle Untersuchungen zeigten, daß der Anstieg des Umfangs des durchluifteten Bereichs von der Düsenkonfiguration abhängt.

3. Das vorliegende mathematische Modell erlaubt die Berechnung der Breite und Höhe der Fackel in Strahlapparaten in Abhängigkeit vom Fluissigkeitsverbrauch und der Strahllänge an der Luft.

\section{Literatur}

[1] Suciu G. D., Smigelschi O/ Chem. Eng. Sci. 1976. V.31. N12. P.1217-1220.

[2] Yablokova M., Sugak A. Hydrodynamik und Stoffaustausch ... // TOCHT, 1988, ò. 22, ${ }^{1} 6$, S. 734-739 (russ.).

[3] Leuzyanskiy. Dynamik von Flïssigkeiten und Gasen. // Verlag „Die Wissenschaft,, ?. 1973. S.848(russ.)

[4] Schlichting. Die Theorie der Grenzschicht. Verlag „Die Wissenschaft", 1974. S.742 (russ.).

[5] Landau., Lifschitz. Theoretische Physik: das Handbuch. In 10 Bänden. Band VI. Hydrodynamik. 1986. S. 736.

\section{Autoren}

Dr. Ing. Lobov Wladimir Jurjewitsch

Lehrstuhl Graphik für Ingenieure

Dr. Ing. Sugak Alexandr Viktorovitsch Lehrstuhl Technologie und Ausruistung

Dr. Ing. habil. Gontscharow Grigori Michailowitsch Lehrstuhl Technologie und Ausrüstung

Staatliche Technische Universität Jaroslavl Tel. 0070852 / 441530

\section{Übersetzung}

\section{Dr. Olga Rösch}

Leiterin des Sprachenzentrums

an der Technischen Fachhochschule Wildau 